\title{
二元铜团簇催化水煤气变换反应机理的理论研究
}

\author{
任宁宁郭玲* 董晓娜文彩霞 \\ (山西师范大学化学与材料科学学院 山西师范大学现代文理学院 山西临汾 041004)
}

\begin{abstract}
摘要 水煤气变换反应是一个重要的反应体系, 它可以去除 $\mathrm{H}_{2}$ 中少量的 $\mathrm{CO}$ 而被应用在质子膜燃料电池中. 然而关于 水煤气变换的反应机理还存在一定的争议, 为阐明其反应机理, 本文采用密度泛函理论 PBE 方法, 金属元素采用 Lanl2dz 基组, 非金属元素采用 6-311++ G(d,p)基组, 对系列二元铜团簇 $\mathrm{Cu}_{6} \mathrm{TM}(\mathrm{TM}=\mathrm{Co}, \mathrm{Rh}, \mathrm{Ir}, \mathrm{Ni}, \mathrm{Pd}, \mathrm{Pt}, \mathrm{Ag}, \mathrm{Au})$ 催 化水煤气变换反应机理进行了研究. 结果表明: $\mathrm{CO}$ 分子比 $\mathrm{H}_{2} \mathrm{O}$ 分子更容易吸附到团簇上. 水煤气变换反应包括三种反 应机理: 羧基反应机理, 氧化还原反应机理, 甲酸反应机理, 相对应的基元反应分别为 $\mathrm{CO}^{*}+\mathrm{O}^{*} \rightarrow \mathrm{CO}_{2}(\mathrm{~g}), \mathrm{CO}^{*}+\mathrm{OH}^{*}$ $\rightarrow \mathrm{COOH}^{*} \rightarrow \mathrm{CO}_{2}(\mathrm{~g})+\mathrm{H}^{*}$, 和 $\mathrm{CO}^{*}+\mathrm{H}^{*}+\mathrm{O}^{*} \rightarrow \mathrm{CHO}^{*}+\mathrm{O}^{*} \rightarrow \mathrm{HCOO}^{* *} \rightarrow \mathrm{CO}_{2}(\mathrm{~g})+\mathrm{H}^{*}$. 甲酸根是实验中最可能检测到的 中间物, 这是由于生成甲酸根有较低的能垒以及甲酸根解离有较高的解离能. $\mathrm{Co}, \mathrm{Rh}, \mathrm{Ni}, \mathrm{Pd}$ 掺杂在 $\mathrm{Cu}_{7}$ 团簇中对水煤气 转化反应的催化效果明显比纯 $\mathrm{Cu}_{7}$ 团簇催化效果好. 采用 $\mathrm{CO}$ 的初始消耗率以及最终 $\mathrm{CO}_{2}$ 的产率进一步研究了在 $\mathrm{Cu}_{6} \mathrm{TM}(\mathrm{TM}=\mathrm{Co}, \mathrm{Rh}, \mathrm{Ni}, \mathrm{Pd})$ 表面甲酸根是反应过程中的旁观者还是一种重要的中间物. 计算结果还表明, 对于 $\mathrm{Cu}_{6} \mathrm{TM}$ $(\mathrm{TM}=\mathrm{Ni}, \mathrm{Pd})$, 由于 $\mathrm{CO}$ 较低的反应能垒, 水煤气变换反应主要按照氧化还原反应机理进行反应, 而对于 $\mathrm{Cu}_{6} \mathrm{TM}(\mathrm{TM}=$ $\mathrm{Co}, \mathrm{Rh})$, 水煤气变换反应三种反应机理均可进行反应. 本文的结果有助于理解水煤气变换反应和设计更好的催化剂.

关键词 密度泛函理论; 水煤气变换反应; 机理; 二元铜团簇
\end{abstract}

\section{Theoretical Study on Menchanism of Water-Gas Shift Reaction Catalyzed by Binary Copper Cluster}

\author{
Ren, Ningning Guo, Ling* Dong, Xiaona Wen, Caixia \\ (School of Chemistry and Material Science, Shanxi Normal University; Shanxi Normal University of Modern Arts \\ and Science, Linfen 041004, Shanxi Province, China)
}

\begin{abstract}
The water-gas shift reaction (WGSR) is an important reaction system and can be applied for removing small amounts of $\mathrm{CO}$ from $\mathrm{H}_{2}$-rich gases for polymer electrolyte membrane fuel cells. However, the mechanism of the reaction is still in dispute. In order to clarify the mechanism of WGSR, the detailed mechanisms of WGSR on a series of binary clusters $\mathrm{Cu}_{6} \mathrm{TM}(\mathrm{TM}=\mathrm{Co}, \mathrm{Rh}, \mathrm{Ir}, \mathrm{Ni}, \mathrm{Pd}, \mathrm{Pt}, \mathrm{Ag}, \mathrm{Au})$ were investigated by density functional theory, using the PBE functional along with the Lanl2dz basis for metals and $6-311++\mathrm{G}(\mathrm{d}, \mathrm{p})$ for non-metals in this paper. The computational results indicated that the absorption of $\mathrm{CO}$ molecules on $\mathrm{Cu}_{6} \mathrm{TM}$ is easier than that of $\mathrm{H}_{2} \mathrm{O}$. WGSR mechanism involves the redox, carboxyl and formate pathways, which correspond to $\mathrm{CO}^{*}+\mathrm{O}^{*} \rightarrow \mathrm{CO}_{2}(\mathrm{~g}), \mathrm{CO}^{*}+\mathrm{OH}^{*} \rightarrow \mathrm{COOH}^{*} \rightarrow \mathrm{CO}_{2}(\mathrm{~g})+\mathrm{H}^{*}$, and $\mathrm{CO}^{*}+\mathrm{H}^{*}+\mathrm{O}^{*} \rightarrow$ $\mathrm{CHO}^{*}+\mathrm{O}^{*} \rightarrow \mathrm{HCOO}^{* *} \rightarrow \mathrm{CO}_{2}(\mathrm{~g})+\mathrm{H}^{*}$, respectively. The experimentally most observed formate can be attributed to its lower formation and higher dissociation barriers. And dopant $\mathrm{Co}, \mathrm{Rh}, \mathrm{Ni}$ and $\mathrm{Pd}$ on copper cluster can have more beneficial effects than pure copper on the catalytic activity. Furthermore, the role of formate, a spectator or key intermediate, on $\mathrm{Cu}_{6} \mathrm{TM}(\mathrm{TM}=$ $\mathrm{Co}, \mathrm{Rh}, \mathrm{Ni}, \mathrm{Pd})$ surfaces has been investigated. WGSR activity has been determined from the initial CO consumption and final $\mathrm{CO}_{2}$ product rates. The calculation results show that WGSR is mostly follows the redox pathway on $\mathrm{Cu}_{6} \mathrm{TM}(\mathrm{TM}=\mathrm{Ni}$, Pd) surface due to the lower CO oxidation barriers; on the other hand, all the three pathways contribute similarly in WGSR on $\mathrm{Cu}_{6} \mathrm{TM}(\mathrm{TM}=\mathrm{Co}, \mathrm{Rh})$ surfaces. The results can help us to understand the catalytic behavior in experiment, design better catalysts, and, therefore, move one step forward to enable hydrogen economy to the practical application.

Keywords density functional theory; water-gas shift reaction; mechanism; binary copper cluster
\end{abstract}

\section{1 引言}

水煤气变换反应(简称 WGSR)是一个重要的工业反
应，在燃料电池和工业方面有大量的应用 ${ }^{[1 \sim 3]}$. 水煤气 变换反应的重要性是能很好地除去氢气生产过程的副 产物 $\mathrm{CO}$, 并且产生额外的氢气. 因此在实验和理论上

*E-mail: 840080528@qq.com; Fax: 0357-2398380

Received November 18, 2014; published January 28, 2015

Supporting information for this article is available free of charge via the Internet at http://sioc-journal.cn

Project supported by the Natural Science Foundation of Shanxi Province (Grant No. 2013011009-6), the High School 131 Leading Talent Project of Shanxi and Training Programs for Innovation and Entrepreneurship of Shanxi Province (No. 2015537).

项目受山西省自然科学基金(No. 2013011009-6), 山西省高等学校 131 领军人才工程项目和山西省高等学校大学生创新创业训练项目(No. 2015537)资 助. 
已经有大量研究报道来阐明水煤气变换反应的反应机

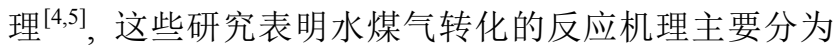
三种: 氧化还原机理, 羧基机理和甲酸机理. Mavrikakis 等 ${ }^{[6]}$ 采用密度泛函理论研究了水煤气变换反应在 $\mathrm{Cu}(111)$ 上的两种反应机理: 氧化还原机理和羧基机理, 计算结果表明羧基机理为主要反应路径. Wang 等 ${ }^{[7]}$ 系统 地研究了水煤气变换反应在几种过渡金属表面的反应, 计算结果表明，水煤气变换反应在 $\mathrm{Cu}(111) 、 \mathrm{Au}(111)$ 、 $\operatorname{Pt}(111)$ 进行有较低的反应能垒, 并且发现在 $\mathrm{Au}(111)$ 表 面主要按照氧化还原机理进行, 然而在 $\mathrm{Cu}(111)$ 和 $\operatorname{Pt}(111)$ 表面三种反应机理均可进行. Vlachos 等 ${ }^{[8]}$ 报道了 水煤气变换反应在 $\mathrm{Ni}(211)$ 和 $\mathrm{Ni}(111)$ 上进行的三种反应 机理, 研究发现在 $\mathrm{Ni}(111)$ 表面, 水煤气变换反应按照羧 基反应机理进行; 而在 $\mathrm{Ni}(211)$ 面上反应按照羧基和甲 酸机理进行反应.

文献中关于水煤气变换的反应机理中, 氧化还原机 理和羧基机理是人们比较认可的反应机理, 然而关于甲 酸机理还存在一定的争论. 在实验上, Apesteguía 等采 用原位红外技术研究水煤气变换反应, 认为甲酸是实验 过程中最容易观测到的一种中间物 ${ }^{[9]}$. 然而, 一些研 究 ${ }^{[10,11]}$ 认为在甲酸机理中由于 $\mathrm{C}-\mathrm{H}$ 键的形成需要很大 的能垒而不可能通过甲酸机理进行反应. 因此, 为进一 步阐明水煤气变换反应机理, 本文系统地研究了不同过 渡金属掺杂铜团簇 $\mathrm{Cu}_{6} \mathrm{TM}(\mathrm{TM}=\mathrm{Co}, \mathrm{Rh}, \mathrm{Ir}, \mathrm{Ni}, \mathrm{Pd}, \mathrm{Pt}$, $\mathrm{Ag}, \mathrm{Au}$ )催化水煤气变换反应机理, 比较不同过渡金属 掺杂对铜团簇催化效果的影响. 希望文本的研究有助于 理解水煤气转化在其他合金上的反应.

\section{2 计算方法}

本文中所有计算都采用 Gaussian09 程序 ${ }^{[12]}$ 中的密 度泛函 PBE 方法, $\mathrm{C}, \mathrm{H}, \mathrm{O}$ 原子采用考虑极化函数和弥 散函数的基组 6-311++ G(d,p), 金属原子采用赝势基组 LANL2DZ ${ }^{[13]}$ 进行构型优化. 运用振动分析获得零点振 动能并确定反应物、中间物以及产物没有虚频, 而过渡 态有唯一虚频, 为势能面上的鞍点. 过渡态构型优化采 用 QST 方法 ${ }^{[14]}$. 所有过渡态采用内禀反应坐标 IRC $^{[15]}$ 分析确认与正确的中间体相连. 自然电荷用 Gaussian09 内置自然键轨道分析程序 NBO 完成. 为验证所选方法
的可靠性，在 PBE/6-311+ $+\mathrm{G}(\mathrm{d}, \mathrm{p})$ 水平上计算 $\mathrm{H}_{2}, \mathrm{CO}$, $\mathrm{CO}_{2}$ 的键长分别为 $0.75,1.14,1.17 \AA$, 相应的实验值 ${ }^{[16,17]}$ 分别为 $0.73,1.14,1.16 \AA$. 计算值与实验值相一致, 说 明本文中所选的方法与基组可靠.

$\mathrm{CO}$ 与 $\mathrm{H}_{2} \mathrm{O}$ 在团簇上的吸附能 $E_{\mathrm{ads}}$ 定义为: $E_{\mathrm{ads}}=$

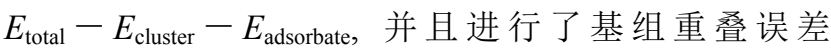
(BSSE) 校正, 其中 $E_{\mathrm{ads}}$ 为团簇吸附后体系的总能量, $E_{\text {cluster }}$ 和 $E_{\text {adsorbate }}$ 分别为团簇和吸附物的能量. $E_{\mathrm{ads}}$ 为负值 表示吸附体系的稳定性.

\section{3 结果与讨论}

\subsection{WGSR 反应机理}

表 1 中列出了水煤气转化反应的三种反应机理的详 细过程. 三种反应机理中首先都是 $\mathrm{CO}$ 和 $\mathrm{H}_{2} \mathrm{O}$ 共吸附到 团簇上, 接着 $\mathrm{H}_{2} \mathrm{O}$ 分子解离成 $\mathrm{H}$ 原子和 $\mathrm{OH}$. 对于氧化 还原机理，接着 $\mathrm{OH}$ 继续解离成 $\mathrm{H}$ 原子和 $\mathrm{O}$ 原子, $\mathrm{CO}$ 被 解离的 $\mathrm{O}$ 原子氧化成 $\mathrm{CO}_{2}$. 在羧基机理中, $\mathrm{H}_{2} \mathrm{O}$ 分子经 过一步解离后，产生的 $\mathrm{OH}$ 与 $\mathrm{CO}$ 反应生成 $\mathrm{COOH}$ 中间 物, 然后 $\mathrm{COOH}$ 中的 $\mathrm{H}$ 原子解离生成 $\mathrm{CO}_{2}$. 在甲酸机理 中, $\mathrm{H}_{2} \mathrm{O}$ 分子经过两步完全解离后 $\mathrm{CO}$ 和 $\mathrm{H}$ 原子反应生 成 $\mathrm{CHO}$ 中间物, $\mathrm{CHO}$ 接着与 $\mathrm{O}$ 原子反应生成甲酸根 (HCOO)中间物, 甲酸根通过两个 $\mathrm{O}$ 原子连接在团簇表 面, 然后 $\mathrm{HCOO}$ 中的 $\mathrm{H}$ 原子解离生成 $\mathrm{CO}_{2}$. 最后团簇表 面剩余的两个 $\mathrm{H}$ 原子结合成 $\mathrm{H}_{2}$.

\section{$3.2 \mathrm{CO}$ 与 $\mathrm{H}_{2} \mathrm{O}$ 吸附结构和吸附能}

首先计算了优化了团簇 $\mathrm{Cu}_{6} \mathrm{TM}(\mathrm{TM}=\mathrm{Co}, \mathrm{Rh}, \mathrm{Ir}$, $\mathrm{Ni}, \mathrm{Pd}, \mathrm{Pt}, \mathrm{Ag}, \mathrm{Au})$ 的稳定结构. Mazalova 等 ${ }^{[18]}$ 已经报道 了 $\mathrm{Cu}_{7}$ 团簇的基态结构为具有 $D_{5 h}$ 对称性的五角双锥结 构, 这种结构包括两种不等价的铜原子: 五元环位点和 顶点. 为保持计算结果的一致性, 使用 PBE 方法重新优 化了 $\mathrm{Cu}_{7}$ 结构. 然后分别用 $\mathrm{TM}$ 替换两种不同的铜原子 进行结构优化. 所有的 $\mathrm{Cu}_{6} \mathrm{TM}$ 基态结构均为五角双雉 构型. 对于 $\mathrm{Cu}_{6} \mathrm{TM}(\mathrm{TM}=\mathrm{Co}, \mathrm{Rh}, \mathrm{Ir}, \mathrm{Ni}, \mathrm{Pd}), \mathrm{TM}$ 原子替 换顶位的铜原子结构比较稳定，它们的对称性均为 $C_{5 v}$, $\mathrm{Cu}_{6} \mathrm{Ni}$ 的结构与报道的相一致 ${ }^{[19]}$. 然而, 对于 $\mathrm{Cu}_{6} \mathrm{TM}$ $(\mathrm{TM}=\mathrm{Pt}, \mathrm{Ag}, \mathrm{Au}), \mathrm{TM}$ 原子替换五元环上的铜原子结构 比较稳定, 对称性分别为 $C_{s}, C_{s}, C_{2 v}$. 在团簇结构优化的

表 $1 \mathrm{Cu}_{6} \mathrm{TM}$ 催化 WGRS 的三种反应机理

Table 1 Three reaction mechanisms for WGRS on $\mathrm{Cu}_{6} \mathrm{TM}$

\begin{tabular}{|c|c|c|}
\hline Redox mechanism & Carboxyl mechanism & Formate mechanism \\
\hline $\mathrm{CO}+* \rightarrow \mathrm{CO}^{*}$ & $\mathrm{CO}+* \rightarrow \mathrm{CO}^{*}$ & $\mathrm{CO}+* \rightarrow \mathrm{CO}^{*}$ \\
\hline $\mathrm{H}_{2} \mathrm{O}+* \rightarrow \mathrm{H}_{2} \mathrm{O}^{*}$ & $\mathrm{H}_{2} \mathrm{O}+* \rightarrow \mathrm{H}_{2} \mathrm{O} *$ & $\mathrm{H}_{2} \mathrm{O}+* \rightarrow \mathrm{H}_{2} \mathrm{O} *$ \\
\hline $\mathrm{H}_{2} \mathrm{O}^{*}+* \rightarrow \mathrm{H}^{*}+\mathrm{OH}^{*}$ & $\mathrm{H}_{2} \mathrm{O}^{*}+* \rightarrow \mathrm{H}^{*}+\mathrm{OH}^{*}$ & $\mathrm{H}_{2} \mathrm{O}^{*}+* \rightarrow \mathrm{H}^{*}+\mathrm{OH}^{*}$ \\
\hline $\mathrm{OH}^{*}+* \rightarrow \mathrm{O}^{*}+\mathrm{H}^{*}$ & $\mathrm{CO}^{*}+\mathrm{OH}^{*} \rightarrow \mathrm{COOH}^{*}+*$ & $\mathrm{OH}^{*}+* \rightarrow \mathrm{O}^{*}+\mathrm{H}^{*}$ \\
\hline $\mathrm{CO}^{*}+\mathrm{O}^{*} \rightarrow \mathrm{CO}_{2} *+*$ & $\mathrm{COOH}^{*}+* \rightarrow \mathrm{CO}_{2} *+\mathrm{H}^{*}$ & $\mathrm{CO}^{*}+\mathrm{H}^{*} \rightarrow \mathrm{CHO}^{*}$ \\
\hline $\mathrm{CO}_{2} * \rightarrow \mathrm{CO}_{2}+*$ & $\mathrm{CO}_{2} * \rightarrow \mathrm{CO}_{2}+*$ & $\mathrm{CHO}^{*}+\mathrm{O}^{*} \rightarrow \mathrm{HCOO}^{* *}$ \\
\hline $\mathrm{H}^{*}+\mathrm{H}^{*} \rightarrow \mathrm{H}_{2}+2^{*}$ & $\mathrm{H}^{*}+\mathrm{H}^{*} \rightarrow \mathrm{H}_{2}+2^{*}$ & $\mathrm{HCOO}^{* *}+* \rightarrow \mathrm{CO}_{2} * *+\mathrm{H}^{*}$ \\
\hline & & $\mathrm{H}^{*}+\mathrm{H}^{*} \rightarrow \mathrm{H}_{2}+2^{*}$ \\
\hline
\end{tabular}


基础上分别计算了 $\mathrm{CO}$ 和 $\mathrm{H}_{2} \mathrm{O}$ 在团簇上的吸附构型, 相 应稳定构型以及前线轨道图见图 1 和图 2, 相应吸附能 与结构参数列于表 2 .

如图 1 和图 2 所示, $\mathrm{CO}$ 分子更容易通过 $\mathrm{C}$ 原子吸附 在 $\mathrm{TM}$ 原子上, 而 $\mathrm{H}_{2} \mathrm{O}$ 分子通过 $\mathrm{O}$ 原子吸附到 $\mathrm{Cu}$ 原子 上. 通过比较表 2 中的团簇 $\mathrm{Cu}_{6} \mathrm{TM}$ 对 $\mathrm{CO}$ 和 $\mathrm{H}_{2} \mathrm{O}$ 分子 吸附的结构参数发现, $\mathrm{CO}$ 和 $\mathrm{H}_{2} \mathrm{O}$ 分子吸附到团簇表面 后, $\mathrm{C}$ - $\mathrm{O}$ 键长拉长, 而 $\mathrm{O}-\mathrm{H}$ 键长没有发生变化, $\mathrm{CO}$ 分 子的振动频率比 $\mathrm{H}_{2} \mathrm{O}$ 分子减小的更多, 团簇对 $\mathrm{CO}$ 的吸 附能明显大于 $\mathrm{H}_{2} \mathrm{O}$. 从表 2 中 $\mathrm{NBO}$ 电荷分析, $\mathrm{CO}$ 吸附 到团簇 $\mathrm{Cu}_{6} \mathrm{TM}$ 上后, 电子从 $\mathrm{Cu}_{6} \mathrm{TM}$ 转移到 $\mathrm{CO}$ 分子上, $\mathrm{CO}$ 作为电子接受体, 接受团簇最高占据轨道(HOMO) 上的电子; 而 $\mathrm{H}_{2} \mathrm{O}$ 吸附到团簇 $\mathrm{Cu}_{6} \mathrm{TM}$ 上后, $\mathrm{H}_{2} \mathrm{O}$ 分子带
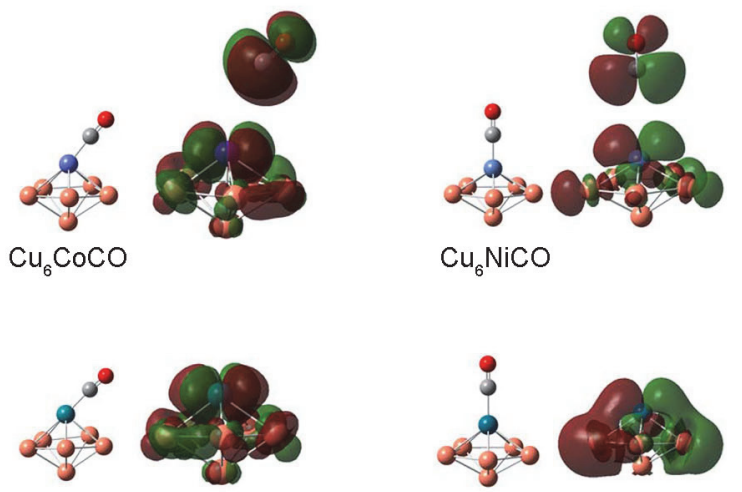

$\mathrm{Cu}_{6} \mathrm{RhCO}$
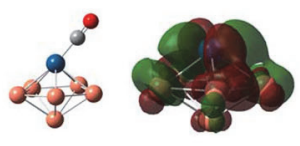

$\mathrm{Cu}_{6} \mathrm{IrCO}$
$\mathrm{Cu}_{6} \mathrm{NiCO}$

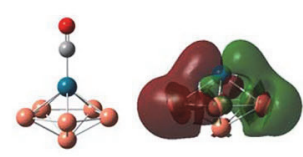

$\mathrm{Cu}_{6} \mathrm{PdCO}$

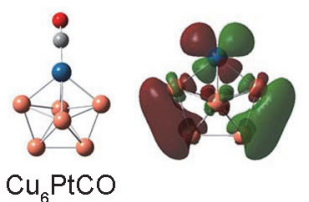

有少量的正电荷，说明电子从 $\mathrm{H}_{2} \mathrm{O}$ 分子转移到才族 $\mathrm{Cu}_{6} \mathrm{TM}$ 上, $\mathrm{H}_{2} \mathrm{O}$ 作为电子供给体, 团簇作为电子接受体, 接受 $\mathrm{H}_{2} \mathrm{O}$ 分子 $\mathrm{HOMO}$ 上的电子. 根据前线轨道理论分 析, CO 的最低空轨道(LUMO)与团簇的 HOMO, $\mathrm{H}_{2} \mathrm{O}$ 的 $\mathrm{HOMO}$ 与团簇的 LUMO 有最大重叠, 很好地解释了 $\mathrm{CO}$ 和 $\mathrm{H}_{2} \mathrm{O}$ 在团族上的吸附构型.

\subsection{WGSR 在团簇 $\mathrm{Cu}_{6} \mathrm{TM}$ 的反应}

计算了 WGSR 在团簇 $\mathrm{Cu}_{6} \mathrm{TM}$ 上反应机理, $\mathrm{Cu}_{6} \mathrm{Co}$ 的势能面曲线以及中间物和过渡态结构见图 3(因 WGSR 在团簇 $\mathrm{Cu}_{6} \mathrm{TM}$ 上反应势能面相似，其他势能面 图见 Supporting Information)，团簇表面各基元反应的反 应热和反应能垒列于表 3 中.

图 $1 \mathrm{CO}$ 吸附到 $\mathrm{Cu}_{6} \mathrm{TM}$ 的稳定结构以及 $\mathrm{CO}$ 的 $\mathrm{LUMO}$ 和团簇 $\mathrm{Cu}_{6} \mathrm{TM}$ 的 $\mathrm{HOMO}$

Figure 1 Optimized structures of $\mathrm{CO}$ adsorbed on $\mathrm{Cu}_{6} \mathrm{TM}$ and $\mathrm{LUMO}$ of $\mathrm{CO}, \mathrm{HOMO}$ of $\mathrm{Cu}_{6} \mathrm{TM}$

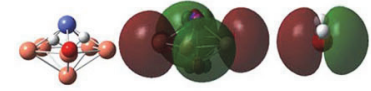

$\mathrm{Cu}_{6} \mathrm{CoH}_{2} \mathrm{O}$
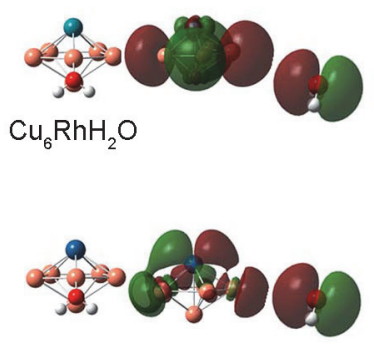

$\mathrm{Cu}_{6} \mathrm{rH}_{2} \mathrm{O}$

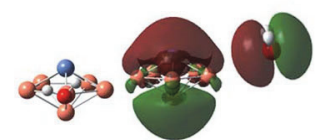

$\mathrm{Cu}_{6} \mathrm{NiH}_{2} \mathrm{O}$
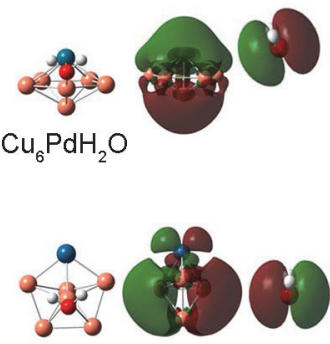

$\mathrm{Cu}_{6} \mathrm{PtH}_{2} \mathrm{O}$

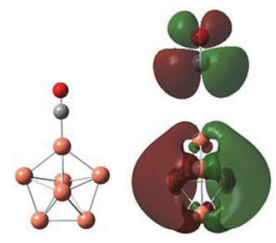

$\mathrm{Cu}_{7} \mathrm{CO}$
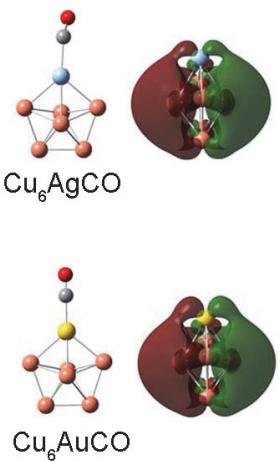

图 $2 \mathrm{H}_{2} \mathrm{O}$ 吸附到 $\mathrm{Cu}_{6} \mathrm{TM}$ 的稳定结构以及 $\mathrm{H}_{2} \mathrm{O}$ 的 HOMO 和团簇 $\mathrm{Cu}_{6} \mathrm{TM}$ 的 LUMO

Figure 2 Optimized structures of $\mathrm{H}_{2} \mathrm{O}$ adsorbed on $\mathrm{Cu}_{6} \mathrm{TM}$ and $\mathrm{HOMO}$ of $\mathrm{H}_{2} \mathrm{O}$, LUMO of $\mathrm{Cu}_{6} \mathrm{TM}$ 
表 $2 \mathrm{CO}$ 和 $\mathrm{H}_{2} \mathrm{O}$ 在团簇 $\mathrm{Cu}_{6} \mathrm{TM}$ 上的吸附能及结构参数

Table 2 Adsorption energies and structure parameters of $\mathrm{CO}$ and $\mathrm{H}_{2} \mathrm{O}$ adsorption on $\mathrm{Cu}_{6} \mathrm{TM}$

\begin{tabular}{|c|c|c|c|c|c|c|c|c|c|c|c|}
\hline Complexes & $\mathrm{M}$ & $d_{\mathrm{C}-\mathrm{O}}$ & $E_{\text {ads }(\mathrm{CO})}$ & $\omega_{\mathrm{CO}}$ & $q_{\mathrm{CO}}$ & Complexes & $\mathrm{M}$ & $d_{\mathrm{H}^{-} \mathrm{O}}$ & $E_{\text {ads }(\mathrm{H} 2 \mathrm{O})}$ & $\omega_{\mathrm{H} 2 \mathrm{O}}$ & $q_{\mathrm{H} 2 \mathrm{O}}$ \\
\hline $\mathrm{Cu}_{6} \mathrm{CoCO}$ & 4 & 1.16 & -1.01 & 1970 & -0.244 & $\mathrm{Cu}_{6} \mathrm{CoH}_{2} \mathrm{O}$ & 4 & 0.97 & -0.36 & $1562 / 3675 / 3784$ & 0.038 \\
\hline $\mathrm{Cu}_{6} \mathrm{NiCO}$ & 1 & 1.16 & -2.24 & 1998 & -0.160 & $\mathrm{Cu}_{6} \mathrm{NiH}_{2} \mathrm{O}$ & 1 & 0.97 & -0.30 & $1576 / 3668 / 3759$ & 0.029 \\
\hline $\mathrm{Cu}_{7} \mathrm{CO}$ & 2 & 1.15 & -0.69 & 2043 & -0.142 & $\mathrm{Cu}_{7} \mathrm{H}_{2} \mathrm{O}$ & 2 & 0.97 & -0.24 & $1555 / 3645 / 3756$ & 0.035 \\
\hline $\mathrm{Cu}_{6} \mathrm{RhCO}$ & 4 & 1.16 & -1.00 & 1959 & -0.134 & $\mathrm{Cu}_{6} \mathrm{RhH}_{2} \mathrm{O}$ & 4 & 0.97 & -0.40 & $1564 / 3666 / 3771$ & 0.040 \\
\hline $\mathrm{Cu}_{6} \mathrm{PdCO}$ & 1 & 1.16 & -1.77 & 2004 & -0.102 & $\mathrm{Cu}_{6} \mathrm{PdH}_{2} \mathrm{O}$ & 1 & 0.97 & -0.29 & $1577 / 3654 / 3779$ & 0.029 \\
\hline $\mathrm{Cu}_{6} \mathrm{AgCO}$ & 2 & 1.15 & -0.30 & 2040 & -0.081 & $\mathrm{Cu}_{6} \mathrm{AgH}_{2} \mathrm{O}$ & 2 & 0.97 & -0.24 & $1556 / 3645 / 3744$ & 0.028 \\
\hline $\mathrm{Cu}_{6} \mathrm{IrCO}$ & 4 & 1.17 & -1.29 & 1949 & -0.223 & $\mathrm{Cu}_{6} \mathrm{IrH}_{2} \mathrm{O}$ & 4 & 0.97 & -0.42 & $1562 / 3665 / 3771$ & 0.046 \\
\hline $\mathrm{Cu}_{6} \mathrm{PtCO}$ & 1 & 1.16 & -1.78 & 2002 & -0.098 & $\mathrm{Cu}_{6} \mathrm{PtH}_{2} \mathrm{O}$ & 1 & 0.97 & -0.45 & $1575 / 3679 / 3759$ & 0.048 \\
\hline $\mathrm{Cu}_{6} \mathrm{AuCO}$ & 2 & 1.15 & -0.49 & 2027 & -0.152 & $\mathrm{Cu}_{6} \mathrm{AuH}_{2} \mathrm{O}$ & 2 & 0.98 & -0.28 & $1558 / 3614 / 3734$ & 0.030 \\
\hline
\end{tabular}

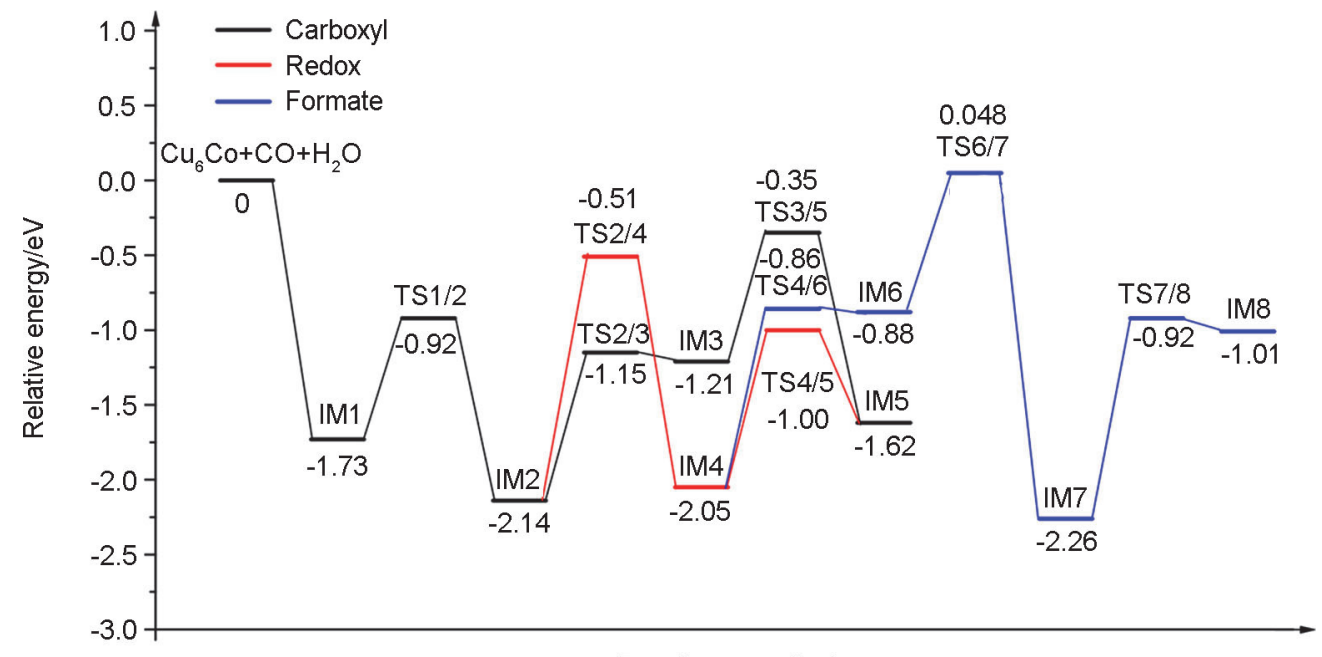

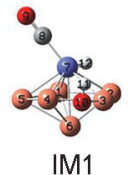
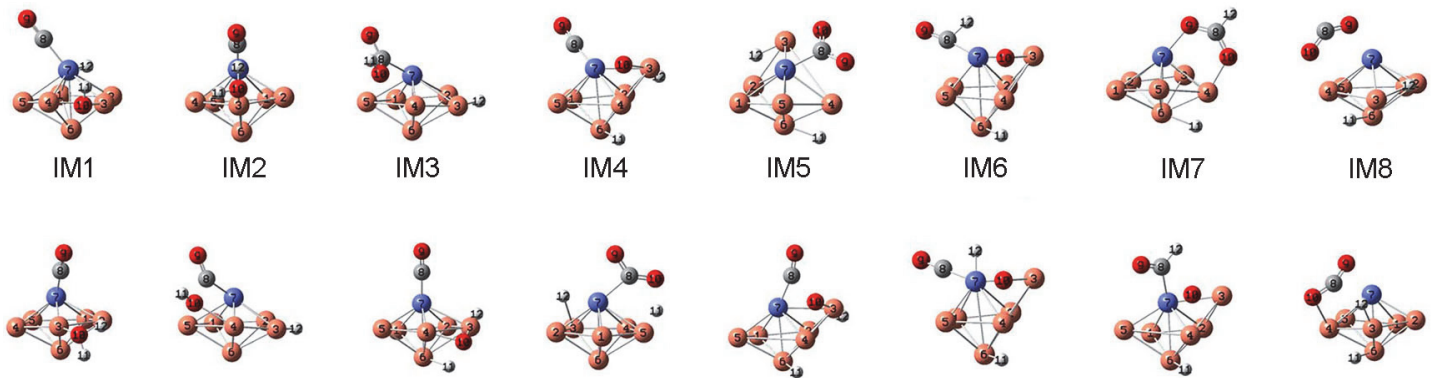

TS3/5

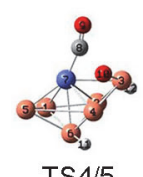

TS4/5

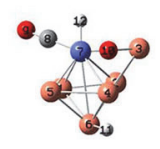

TS4/6

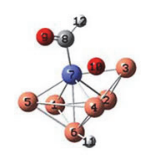

TS6/7

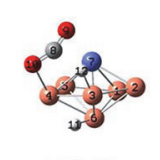

$\mathrm{TS} 7 / 8$

图 3 WGSR 在 $\mathrm{Cu}_{6} \mathrm{Co}$ 上的势能面曲线以及中间物和过渡态的优化结构

Figure 3 Potential energy profiles for WGSR on $\mathrm{Cu}_{6} \mathrm{Co}$ and the optimized structures of intermediates and transition states

如图 3 所示, 反应是从 $\mathrm{CO}$ 和 $\mathrm{H}_{2} \mathrm{O}$ 共吸附到 $\mathrm{Cu}_{6} \mathrm{Co}$ 形成 IM1 开始的, 它们的共吸附能为 $1.73 \mathrm{eV}$. 接着经过 过渡态 $\mathrm{TS} 1 / 2$ 水解离为 $\mathrm{H}$ 和 $\mathrm{OH}$ 形成 IM2, 水解离所需 要的能垒为 $0.81 \mathrm{eV}$, 放出热量 $0.41 \mathrm{eV}$. 在 $\mathrm{TS} 1 / 2$ 中, $\mathrm{H}$ 原子和 $\mathrm{OH}$ 中 $\mathrm{O}$ 原子的距离为 $1.71 \AA$, 大于水分子中的 $\mathrm{O}-\mathrm{H}$ 键长. 水分子解离后, 接下来的反应路径分为羧 基反应机理和氧化还原反应机理. 在羧基反应机理, $\mathrm{IM} 2$ 中 $\mathrm{CO}$ 和 $\mathrm{OH}$ 相互靠近形成具有羧基 $(\mathrm{COOH})$ 结构的 IM 3 , 从 IM2 到 IM 3 经过过渡态 TS $2 / 3$, 在过渡态 TS $2 / 3$
中, $\mathrm{C}$ 原子与 $\mathrm{OH}$ 中 $\mathrm{O}$ 原子的距离仅为 $1.67 \AA$, 说明一个 新的 $\mathrm{C}-\mathrm{O}$ 键将形成. 这步反应为吸热反应, 反应热为 $0.93 \mathrm{eV}$, 反应所需的活化能为 $0.99 \mathrm{eV}$. 接着, IM3 中羧 基中 $\mathrm{O}-\mathrm{H}$ 键断裂产生 $\mathrm{CO}_{2}$ 分子和 $\mathrm{H}$ 原子, $\mathrm{H}-\mathrm{O}$ 键断 裂所需要的能垒为 $0.86 \mathrm{eV}$. 在 TS3/5 中, 断裂的 $\mathrm{H}$ 原子 和 $\mathrm{O}$ 原子之间的距离为 $1.48 \AA$. 在羧基反应机理中, IM 2 到 IM3 为反应的决速步.

在氧化还原反应机理中, 水分子一次解离后形成的 $\mathrm{OH}$ 经过 TS2/4 继续解离形成一个具有游离的 $\mathrm{O}$ 原子的 
表 3 团簇表面各基元反应的反应热和反应能

Table 3 Reaction energies $(\Delta H)$ and activation barriers $\left(E_{\mathrm{a}}\right)$, in $\mathrm{eV}$, of elementary steps on clusters

\begin{tabular}{|c|c|c|c|c|c|c|c|c|c|c|}
\hline & & $\mathrm{Co}$ & $\mathrm{Ni}$ & $\mathrm{Cu}$ & $\mathrm{Rh}$ & $\mathrm{Pd}$ & $\mathrm{Ag}$ & $\mathrm{Ir}$ & $\mathrm{Pt}$ & $\mathrm{Au}$ \\
\hline \multirow[t]{2}{*}{$\mathrm{IM} 1 \rightarrow \mathrm{TS} 1 / 2 \rightarrow \mathrm{IM} 2$} & $\Delta H_{1 / 2}$ & -0.41 & 0.78 & 0.22 & -0.32 & 0.84 & 0.47 & -0.57 & 0.68 & 0.68 \\
\hline & $E_{\mathrm{a} 1 / 2}$ & 0.81 & 0.82 & 1.09 & 0.64 & 0.97 & 1.22 & 0.49 & 0.99 & 1.23 \\
\hline \multirow[t]{2}{*}{$\mathrm{IM} 2 \rightarrow \mathrm{TS} 2 / 3 \rightarrow \mathrm{IM} 3$} & $\Delta H_{2 / 3}$ & 0.93 & 0.43 & 0.04 & 0.83 & 0.33 & -0.14 & 1.13 & -0.03 & -0.27 \\
\hline & $E_{\mathrm{a} 2 / 3}$ & 0.99 & 0.44 & 0.38 & 1.01 & 0.54 & 0.26 & 1.82 & 0.09 & 0.20 \\
\hline $\mathrm{IM} 2 \rightarrow \mathrm{TS} 2 / 4 \rightarrow \mathrm{IM} 4$ & $E_{\mathrm{a} 2 / 4}$ & 1.63 & 1.50 & 1.59 & 1.51 & 1.78 & 1.72 & 1.72 & 1.58 & 1.88 \\
\hline \multirow[t]{2}{*}{$\mathrm{IM} 3 \rightarrow \mathrm{TS} 3 / 5 \rightarrow \mathrm{IM} 5$} & $\Delta H_{3 / 5}$ & -0.41 & 0.12 & -0.10 & -0.34 & 0.21 & -0.10 & -0.20 & 0.47 & -0.06 \\
\hline & $E_{\mathrm{a} 3 / 5}$ & 0.86 & 0.92 & 0.92 & 0.86 & 0.97 & 1.03 & 0.77 & 1.31 & 1.12 \\
\hline \multirow[t]{2}{*}{$\mathrm{IM} 4 \rightarrow \mathrm{TS} 4 / 5 \rightarrow \mathrm{IM} 5$} & $\Delta H_{4 / 5}$ & 0.43 & -0.37 & -0.40 & 0.05 & -0.56 & -0.67 & 0.38 & -0.12 & -0.74 \\
\hline & $E_{\mathrm{a} 4 / 5}$ & 1.05 & 0.16 & 0.34 & 0.95 & 0.06 & 0.13 & 1.19 & 0.17 & 0.22 \\
\hline \multirow[t]{2}{*}{$\mathrm{IM} 4 \rightarrow \mathrm{TS} 4 / 6 \rightarrow \mathrm{IM} 6$} & $\Delta H_{4 / 6}$ & 1.17 & 0.61 & 0.60 & 0.98 & 0.49 & 0.62 & 1.10 & 0.60 & 0.51 \\
\hline & $E_{\mathrm{a} 4 / 6}$ & 1.19 & 0.74 & 1.06 & 1.05 & 0.59 & 0.75 & 1.22 & 1.27 & 0.74 \\
\hline \multirow[t]{2}{*}{$\mathrm{IM} 6 \rightarrow \mathrm{TS} 6 / 7 \rightarrow \mathrm{IM} 7$} & $\Delta H_{6 / 7}$ & -1.38 & -1.91 & -2.09 & -1.11 & -2.20 & -2.35 & -0.52 & -1.64 & -1.94 \\
\hline & $E_{\mathrm{a} 6 / 7}$ & 0.93 & 0.22 & 0.71 & 0.74 & 0.16 & 0.75 & 1.09 & 0.78 & 0.68 \\
\hline \multirow[t]{2}{*}{$\mathrm{IM} 7 \rightarrow \mathrm{TS} 7 / 8 \rightarrow \mathrm{IM} 8$} & $\Delta H_{7 / 8}$ & 1.25 & 0.82 & 1.44 & 1.03 & 0.50 & 0.81 & 0.51 & 0.28 & 0.22 \\
\hline & $E_{\mathrm{a} 7 / 8}$ & 1.34 & 1.02 & 1.57 & 1.10 & 0.90 & 0.96 & 0.95 & 1.04 & 0.69 \\
\hline
\end{tabular}

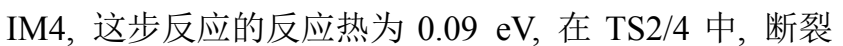
的 $\mathrm{H}-\mathrm{O}$ 键长拉长为 $1.80 \AA$, 从 $\mathrm{IM} 2$ 到 IM4 反应活化能 为 $1.63 \mathrm{eV}$, 这步反应为该条反应路径的决速步. 接着 $\mathrm{IM} 4$ 中 $\mathrm{CO}$ 与解离的 $\mathrm{O}$ 原子经过 $\mathrm{TS} 4 / 5$ 生成 $\mathrm{CO}_{2}$, 在 $\mathrm{TS} 4 / 5$ 中, $\mathrm{CO}$ 中 $\mathrm{C}$ 原子与 $\mathrm{O}$ 原子的的距离仅为 $1.82 \AA$, 说明一个新的 $\mathrm{C}-\mathrm{O}$ 键将形成.

在甲酸机理中, 水分子经过两次解离成 $\mathrm{O}$ 原子和两 个 $\mathrm{H}$ 原子后, $\mathrm{CO}$ 与一个 $\mathrm{H}$ 原子相互作用生成一个 $\mathrm{CHO}$ 中间物 IM6, 这步反应为吸热反应, 反应热分别为 1.17 $\mathrm{eV}$, 反应活化能为 $1.19 \mathrm{eV}$. 接着, $\mathrm{CHO}$ 中的 $\mathrm{C}$ 原子与剩 余的 $\mathrm{O}$ 原子相互靠近, 形成 $\mathrm{HCOO}$ 中间物 IM7, HCOO 通过两个 $\mathrm{O}$ 原子连接在 $\mathrm{Cu}_{6} \mathrm{Co}$ 表面, 从 IM6 到 IM7 经 过过渡态 TS6/7, 反应活化能为 $0.93 \mathrm{eV}$. IM4 到 IM6 为 吸热反应, IM6 到 IM7 为放热反应, 从热力学观点说明 $\mathrm{CHO}^{*}$ 不是一个稳定的中间物, 极易生成稳定的中间物 $\mathrm{HCOO}^{* *}$. 接着, IM7 中甲酸根中 $\mathrm{C}-\mathrm{H}$ 键断裂产生 $\mathrm{CO}_{2}$ 分子和 $\mathrm{H}$ 原子, $\mathrm{C}-\mathrm{H}$ 键断裂所需要的能垒为 $1.34 \mathrm{eV}$. 在 TS7/8 中, 断裂的 $\mathrm{C}$ 原子和 $\mathrm{H}$ 原子之间的距离分别为 $1.92 \AA, \mathrm{C}-\mathrm{H}$ 键有唯一虚频为 $-305.91 \mathrm{~cm}^{-1}$. 最后 $\mathrm{CO}_{2}$ 和 $\mathrm{H}$ 原子从团簇 $\mathrm{Cu}_{6} \mathrm{Co}$ 上解离标志着反应完成.

比较表 3 中数据发现, $\mathrm{Cu}_{6} \mathrm{Co}, \mathrm{Cu}_{6} \mathrm{Rh}, \mathrm{Cu}_{6} \mathrm{Ni}, \mathrm{Cu}_{6} \mathrm{Pd}$ 团簇对 WGSR 有较好的催化活性. 这是由于金属原子 $\mathrm{TM}$ 掺杂在 $\mathrm{Cu}_{7}$ 团簇后, 团族的 $\mathrm{d}$ 电子的平均能量向费 米能级移动, 从而提高了团簇的催化活性. 但是 $\mathrm{d}$ 电子 的平均能量并不是判断过渡金属催化活性的唯一标准, 反应过程中团簇对中间物的吸附能力也决定了团簇的 催化活性 ${ }^{[20]}$. $\mathrm{Cu}_{6} \mathrm{Ir}$ 在反应 IM2 到 IM3 过程中, 由于
$\mathrm{Cu}_{6} \mathrm{Ir}$ 对 $\mathrm{OH}$ 有较强的吸附性导致形成中间物 IM3 时需 要较大的能垒, $\mathrm{Cu}_{6} \mathrm{Pt}$ 对 $\mathrm{COOH}$ 的吸附能大于 $\mathrm{Cu}_{7}$ 使得 $\mathrm{COOH}$ 解离出 $\mathrm{H}$ 原子时能垒较大, 对于 $\mathrm{Cu}_{6} \mathrm{Ag}$ 和 $\mathrm{Cu}_{6} \mathrm{Au}$ 对 $\mathrm{H}_{2} \mathrm{O}$ 的吸附能比 $\mathrm{Cu}_{7}$ 大, 使得 $\mathrm{H}_{2} \mathrm{O}$ 分子的解离过程比 较困难, 所以 $\mathrm{Cu}_{6} \mathrm{TM}(\mathrm{TM}=\mathrm{Ir}, \mathrm{Pt}, \mathrm{Ag}, \mathrm{Au})$ 对 WGSR 的催 化活性较低.

为进一步探讨甲酸根是反应过程中的旁观者还是 一种重要的中间物, 比较了 WGSR 在 $\mathrm{Cu}_{6} \mathrm{Co}, \mathrm{Cu}_{6} \mathrm{Rh}$, $\mathrm{Cu}_{6} \mathrm{Ni}, \mathrm{Cu}_{6} \mathrm{Pd}$ 四种团簇表面的三种反应机理. 实验上通 常采用 $\mathrm{CO}$ 的初始消耗率以及最终 $\mathrm{CO}_{2}$ 的产率来评价催 化剂的催化活性. 在三种反应机理中, 消耗 $\mathrm{CO}$ 的反应 步骤分别为 $\mathrm{CO}^{*}+\mathrm{OH}^{*} \rightarrow \mathrm{TS} 2 / 3 \rightarrow \mathrm{COOH}^{*}, \mathrm{CO}^{*}+$ $\mathrm{O}^{*} \rightarrow \mathrm{TS} 4 / 5 \rightarrow \mathrm{CO}_{2}, \mathrm{CO}^{*}+\mathrm{H}^{*} \rightarrow \mathrm{TS} 4 / 6 \rightarrow \mathrm{CHO}^{*}$. 生成的 $\mathrm{CO}_{2}$ 反应为 $\mathrm{COOH}^{*} \rightarrow \mathrm{TS} 3 / 5 \rightarrow \mathrm{CO}_{2}+\mathrm{H}^{*}, \mathrm{CO}^{*}+\mathrm{O}^{*} \rightarrow$ $\mathrm{TS} 4 / 5 \rightarrow \mathrm{CO}_{2}, \mathrm{HCOO}^{* *} \rightarrow \mathrm{TS} 7 / 8 \rightarrow \mathrm{CO}_{2}+\mathrm{H}^{*}$. 理论上 ${ }^{[7]}$ 采 用相应反应的能垒 $E_{2 / 3} / E_{4 / 5}$ 和 $E_{4 / 6} / E_{4 / 5}$ 来比较三种反应机 理的 $\mathrm{CO}$ 初始消耗量, 采用 $E_{3 / 5} / E_{4 / 5}$ 和 $E_{7 / 8} / E_{4 / 5}$ 来比较三 种反应机理的 $\mathrm{CO}_{2}$ 产量. 本文采用这种方法比较了水煤 气转化在 $\mathrm{Cu}_{6} \mathrm{TM}(\mathrm{TM}=\mathrm{Co}, \mathrm{Rh}, \mathrm{Ni}, \mathrm{Pd})$ 的三种反应机理, 比较结果见图 4. 对于 $\mathrm{CO}$ 的相对消耗量如图 5 7 所示, 对于 $\mathrm{Cu}_{6} \mathrm{Co}$ 和 $\mathrm{Cu}_{6} \mathrm{Rh}, E_{2 / 3} / E_{4 / 5}$ 和 $E_{4 / 6} / E_{4 / 5}$ 的比值范围在 0.94 到 1.13 之间. 而对于 $\mathrm{Cu}_{6} \mathrm{Ni}$ 和 $\mathrm{Cu}_{6} \mathrm{Pd}, E_{2 / 3} / E_{4 / 5}$ 和 $E_{4 / 6} / E_{4 / 5}$ 的比值 $2.75 \sim 9.83$. 这说明对于 $\mathrm{Cu}_{6} \mathrm{TM}(\mathrm{TM}=$ $\mathrm{Ni}, \mathrm{Pd}$ ), 消耗 $\mathrm{CO}$ 主要通过氧化还原反应机理; 对于 $\mathrm{Cu}_{6} \mathrm{TM}(\mathrm{TM}=\mathrm{Co}, \mathrm{Rh})$, 三种反应路径对 $\mathrm{CO}$ 的消耗量相 当. 同样对于 $\mathrm{CO}_{2}$ 的相对产量, 对于 $\mathrm{Cu}_{6} \mathrm{TM}(\mathrm{TM}=\mathrm{Co}$, $\mathrm{Rh}), E_{3 / 5} / E_{4 / 5}$ 和 $E_{7 / 8} / E_{4 / 5}$ 的比值在 1 左右, 说明生成 $\mathrm{CO}_{2}$ 
通过三种反应路径，而对于 $\mathrm{Cu}_{6} \mathrm{TM}(\mathrm{TM}=\mathrm{Ni}, \mathrm{Pd})$, $E_{3 / 5} / E_{4 / 5}$ 和 $E_{7 / 8} / E_{4 / 5}$ 都有一个较大的比值, 说明 $\mathrm{CO}_{2}$ 的生 成主要是通过氧化还原反应机理的. 综合以上比较, 对 于 $\mathrm{Cu}_{6} \mathrm{TM}(\mathrm{TM}=\mathrm{Co}, \mathrm{Rh}), \mathrm{WGSR}$ 通过三种反应机理进 行, 而对于 $\mathrm{Cu}_{6} \mathrm{TM}(\mathrm{TM}=\mathrm{Ni}, \mathrm{Pd}), \mathrm{WGSR}$ 主要通过氧化 还原反应机理进行.

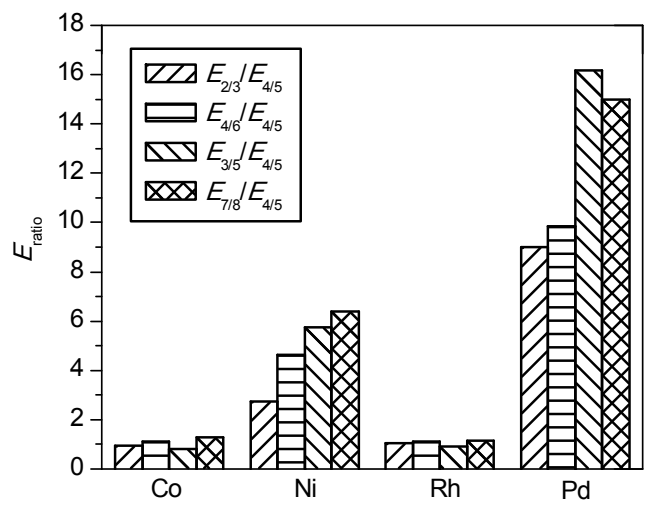

图 4 团簇 $\mathrm{Cu}_{6} \mathrm{TM}(\mathrm{TM}=\mathrm{Co}, \mathrm{Rh}, \mathrm{Ni}, \mathrm{Pd})$ 上 $\mathrm{CO}$ 消耗量 $\left(E_{2 / 3} / E_{4 / 5}\right.$ 和 $\left.E_{4 / 6} / E_{4 / 5}\right)$ 和 $\mathrm{CO}_{2}$ 产量 $\left(E_{3 / 5} / E_{4 / 5}\right.$ 和 $\left.E_{7 / 8} / E_{4 / 5}\right)$ 的活化能比率

Figure 4 The $E_{\mathrm{a}}$ ratio comparison in $\mathrm{CO}$ consumption $\left(E_{2 / 3} / E_{4 / 5}\right.$ and $\left.E_{4 / 6} / E_{4 / 5}\right)$ and $\mathrm{CO}_{2}$ production $\left(E_{3 / /} / E_{4 / 5}\right.$ and $\left.E_{7 / 8} / E_{4 / 5}\right)$ on $\mathrm{Cu}_{6} \mathrm{TM}(\mathrm{TM}=\mathrm{Co}$, $\mathrm{Rh}, \mathrm{Ni}, \mathrm{Pd})$

\section{4 结论}

本文采用密度泛函理论系统地研究了水煤气转化 反应在 $\mathrm{Cu}_{n} \mathrm{TM}(\mathrm{TM}=\mathrm{Co}, \mathrm{Rh}, \mathrm{Ir}, \mathrm{Ni}, \mathrm{Pd}, \mathrm{Pt}, \mathrm{Cu}, \mathrm{Ag}, \mathrm{Au})$ 上的反应机理, 计算体系采用 $\mathrm{PBE}$ 方法, $\mathrm{C}, \mathrm{H}, \mathrm{O}$ 采用 6-311++ G(d,p)基组，金属原子采用 LANL2DZ 赝势基 组. 计算结果表明, 首先, $\mathrm{CO}$ 分子更容易通过 $\mathrm{C}$ 原子吸 附在 $\mathrm{TM}$ 原子上, 而 $\mathrm{H}_{2} \mathrm{O}$ 分子通过 $\mathrm{O}$ 原子吸附到 $\mathrm{Cu}$ 原 子上, 并用前线轨道理论解释了 $\mathrm{CO}$ 和 $\mathrm{H}_{2} \mathrm{O}$ 在团簇上吸 附, 团簇对 $\mathrm{CO}$ 的吸附能明显大于 $\mathrm{H}_{2} \mathrm{O}$. 其次, 水煤气变 换反应包括三种主要的反应机理: 羧基机理, 氧化还原 机理, 甲酸机理. 在着基机理 $\mathrm{CO}^{*}+\mathrm{OH}^{*} \rightarrow \mathrm{COOH}^{*} \rightarrow$ $\mathrm{CO}_{2}(\mathrm{~g})+\mathrm{H}^{*}$ 中包括 $\mathrm{C}-\mathrm{O}$ 键的形成和 $\mathrm{O}-\mathrm{H}$ 键的断裂; 在氧化还原机理中, $\mathrm{CO}$ 与解离的 $\mathrm{O}$ 原子形成 $\mathrm{CO}_{2}$; 在甲 酸机理的基元反应中包括 $\mathrm{C}-\mathrm{H}$ 键和 $\mathrm{O}-\mathrm{H}$ 键的形成以 及 $\mathrm{C}-\mathrm{H}$ 键的断裂. 甲酸是实验中最可能检测到的中间 物, 这是由于生成甲酸根有较低的能垒以及甲酸根解离 有较高的解离能. 最后进一步比较了三种反应机理对于 $\mathrm{Cu}_{6} \mathrm{TM}(\mathrm{TM}=\mathrm{Ni}, \mathrm{Pd})$, 由于 $\mathrm{CO}$ 被 $\mathrm{O}$ 原子氧化具有很低 的能量, 水煤气变换反应主要通过氧化还原反应机理进
行, 对于 $\mathrm{Cu}_{6} \mathrm{TM}(\mathrm{TM}=\mathrm{Co}, \mathrm{Rh})$, 三种机理消耗 $\mathrm{CO}$ 具有 相近的能垒反应可以通过三种路径进行. 希望本章的研 究结果能更好地帮助我们理解水煤气转化的反应机理, 同时对二元合金团簇和催化剂的设计有一定的指导意 义.

\section{References}

[1] Chen, L.; Ni, G.; Han, B.; Zhou, C. G.; Wu, J. P. Acta Chim. Sinica 2011，69，393. (陈否，倪刚，韩波，周成刚，吴金平，化学学报， 2011, 69, 393.)

[2] Zhang, S. R.; Shan, J. J.; Zhu, Y.; Frenkel, A. I.; Patlolla, A.; Huang, W. X.; Yoon, S. J.; Wang, L.; Yoshida, H.; Takeda, S.; Tao, F. J. Am. Chem. Soc. 2013, 135, 8283.

[3] Ammal, S. C.; Heyden, A. ACS Catal. 2014, 4, 3654.

[4] Wang, G. C.; Cui, Y. B.; Sun, Y. H.; Zhong, B. Acta Chim. Sinica 1998, 56, 867. (王贵昌, 崔永斌, 孙予罕, 钟炳, 化学学报, 1998, $56,867$.

[5] Huang, S. C.; Lin, C. H.; Wang, J. H. J. Phys. Chem. C 2010, 114, 9826.

[6] Gokhale, A. A.; Dumesic, J. A.; Mavrikakis, M. J. Am. Chem. Soc. 2008, 130, 1402 .

[7] Lin, C. H.; Chen, C. L.; Wang, J. H. J. Phys. Chem. C 2011, 115, 18582.

[8] Catapan, R. C.; Oliveira, A. A. M.; Chen, Y.; Vlachos, D. G. J. Phys. Chem. C 2012, 116, 20281.

[9] Vignatti, C.; Avila, M. S.; Apesteguía, C. R.; Garetto, T. F. Int. J. Hydrogen Energy 2010, 35, 7302.

[10] Chen, Y. Y.; Dong, M.; Wang, J. G.; Jiao, H. J. J. Phys. Chem. C 2012, 116, 25368.

[11] Wei, H. J.; Gomez, C.; Meyer, R. J. Top. Catal. 2012, 55, 313.

[12] Frisch, M. J.; Trucks, G. W.; Schlegel, H. B.; Scuseria, G. E.; Robb, M. A.; Cheeseman, J. R.; Scalmani, G.; Barone, V.; Mennucci, B.; Petersson, G. A.; Nakatsuji, H.; Caricato, M.; Li, X.; Hratchian, H. P.; Izmaylov, A. F.; Bloino, J.; Zheng, G.; Sonnenberg, J. L.; Hada, M.; Ehara, M.; Toyota, K.; Fukuda, R.; Hasegawa, J.; Ishida, M.; Nakajima, T.; Honda, Y.; Kitao, O.; Nakai, H.; Vreven, T.; Montgomery, J. A.; Peralta, J. E.; Ogliaro, F.; Bearpark, M.; Heyd, J. J.; Brothers, E.; Kudin, K. N.; Staroverov, V. N.; Keith, T.; Kobayashi, R.; Normand, J.; Raghavachari, K.; Rendell, A.; Burant, J. C.; Iyengar, S. S.; Tomasi, J.; Cossi, M.; Rega, N.; Millam, J. M.; Klene, M.; Knox, J. E.; Cross, J. B.; Bakken, V.; Adamo, C.; Jaramillo, J.; Gomperts, R.; Stratmann, R. E.; Yazyev, O.; Austin, A. J.; Cammi, R.; Pomelli, C.; Ochterski, J. W.; Martin, R. L.; Morokuma, K.; Zakrzewski, V. G.; Voth, G. A.; Salvador, P.; Dannenberg, J. J.; Dapprich, S.; Daniels, A. D.; Farkas, O.; Foresman, J. B.; Ortiz, J. V.; Cioslowski, J.; Fox, D. J. Gaussian 09, Revision C. 01, Gaussian Inc., Wallingford, CT, 2010.

[13] Couty, M.; Hall, M. B. J. Comput. Chem. 1996, 17, 1359.

[14] Peng, C. Y.; Ayala, P. Y.; Schlegel, H. B. J. Comput. Chem. 1996, 17, 49.

[15] (a) Gonzalez, C.; Schlegel, H. B. J. Chem. Phys. 1989, 90, 2154. (b) Gonzalez, C.; Schlegel, H. B. J. Phys. Chem. 1990, 54, 5523

[16] Zhao, Y.; Tian, D. X. Comput. Theor. Chem. 2012, 991, 40.

[17] Huber, K. P.; Herzberg, G. Molecular Spectra and Molecular Structure IV. Constants of Diatomic Molecules, Van Nostrand Reinhold, New York, 1979.

[18] Mazalova, V. L.; Soldatov, A. V. J. Phys. Chem. C 2009, 113, 9086.

[19] Han, S. L.; Xue, X. L.; Nie, X. C.; Zhai, H.; Wang, F.; Sun, Q.; Jia, Y.; Li, S. F.; Guo, Z. X. Phys. Lett. A 2010, 374, 4324.

[20] Wang, G. C.; Nakamura, J. J. Phys. Chem. Lett. 2010, 1, 3053.

(Cheng, B.; Fan, Y.) 